\title{
Vivienda social y segregación socioespacial en una ciudad pequeña: el caso de Angol, Chile
}

\section{Luis Vergara-Erices y Alan Garín Contreras}

Universidad de La Frontera, Temuco, Chile.

E-mail: l.vergara002@gmail.com

Resumen: Este trabajo busca reconocer el impacto que la vivienda social ha tenido sobre los procesos de segregación socioespacial en la ciudad de Angol, una ciudad menor. La metodología empleada fue mixta, utilizando el índice de similitud de Duncan, registros de permisos de edificación municipales y entrevistas semiestructuradas a habitantes de conjuntos de vivienda social y agentes con injerencia en el desarrollo urbano local. Los resultados indican que la vivienda social en Angol ha contribuido a aumentar la segregación espacial, sin embargo, su manifestación ha adquirido una escala espacial reducida, que ha permitido que grupos socioeconómicos disímiles habiten en condiciones de proximidad espacial, limitando así el aumento de la segregación y exclusión social tanto funcional como simbólica. Se concluye que el estudio de la manifestación de la segregación socioespacial en las ciudades menores puede contribuir en la generación de políticas públicas que suavicen los efectos evidenciados en las grandes ciudades. Angol.

Palabras clave: globalización, segregación socioespacial, vivienda social,

\section{Social housing and sociospatial segregation in a small town: the case of Angol, Chile}

\begin{abstract}
This work seeks to recognize the impact that social housing has had on the processes of socio-spatial segregation in the city of Angol, a minor city. A mixed methodology was occupied, utilyzing the similarity index of Duncan, municipal building logs and semistructured interviews of residents of social housing and agents with interference in local urban development. The results indicate that social housing in Angol has contributed to increased spatial segregation, however, its expression has acquired a small spatial scale, which has allowed groups inhabit dissimilar socioeconomic conditions of spatial proximity, limiting the increase in segregation and both functional and symbolic social exclusion. We conclude that the study of the manifestation of sociospatial segregation in the smaller cities can contribute to the generation of public policies that may temper the effects evident in large cities. Angol.

Keywords: globalization, socio-spatial segregation, social housing,
\end{abstract}




\title{
Vivenda social e segregação socioespacial numa cidade pequena: o caso de Angol, Chile
}

\begin{abstract}
Resumo: Este trabalho procura reconhecer o impacto que a vivenda social tem tido sobre os processos de segregação socioespacial na cidade de Angol, uma cidade menor. A metodologia empregada foi mista, utilizando o índice de similitude de Duncan, registros de licenças de edificação municipais e entrevistas semiestruturadas para habitantes de conjuntos de vivenda social e agentes com ingerência no desenvolvimento urbano local. Os resultados indicam que a vivenda social em Angol tem contribuído para aumentar a segregação espacial, porém, sua manifestação tem adquirido uma escala espacial reduzida, que há permitido que grupos socioeconómicos diferentes habitassem em condiciones de proximidade espacial, limitando assim o aumento da segregação e exclusão social tanto funcional como simbólica. Conclui-se que o estudo da manifestação da segregação socioespacial nas cidades menores pode contribuir na geração de políticas públicas que suavizem os efeitos evidenciados nas grandes cidades.
\end{abstract} Angol.

Palavras-Chave: globalização, segregação socioespacial, vivenda social,

Transformaciones de la segregación socioespacial en la era neoliberal

En la década del setenta del siglo veinte, la economía internacional evidenció una serie de transformaciones, las que dieron lugar al desarrollo de una nueva fase del capitalismo que ha sido denominada como postfordismo o globalización. Una de las principales mutaciones fue un cambio en "la configuración de los procesos de acumulación de capital caracterizado por su transferencia de los países de renta elevada a los espacios de renta baja, y al mismo tiempo, una crisis estructural del modelo de acumulación fordista y del dogma económico prevaleciente hasta entonces: el keynesianismo” (Arteaga, 2005: 349).

Los cambios, asociados a la globalización o capitalismo neoliberal, han llevado a una mayor polarización mundial, tanto entre países como al interior de los mismos. En este sentido Escobar de Pavón (2005:58) menciona que "mientras se interrelacionan ciertas partes del mundo y regiones o sectores dentro de los países, se margina o excluye a otros, limitando sus posibilidades para desarrollarse y cambiar su ubicación dentro del sistema”.

En América Latina, la crisis de la deuda permite la aplicación de las políticas de ajuste estructural con el objeto de insertar las economías en un nuevo modelo internacional globalizado, que desde los organismos multilaterales se plantea como la única alternativa de desarrollo (Ugarteche, 2000). Los resultados de este ajuste y cambio en los modelos de desarrollo vigentes, provocaron un aumento en los niveles de pobreza, segregación socioespacial y desigualdad social, caracterizado por la existencia de un desempleo creciente, empleos de baja calidad y disminución de los salarios.

Por su parte, la segregación socioespacial como problema social, se 
agudizó en los últimos años al igual que sus consecuencias negativas, especialmente para los más pobres (Suárez, 2005). En esta dirección, Valdés y Koch (2009:90) indican que la segregación socioespacial es tanto un:

"Fenómeno de creciente expansión en las ciudades de los países desarrollados como en los emergentes y se relacionan con nuevas formas de desigualdades sociales que afectan a las grandes metrópolis. Estas transformaciones tienen su punto de partida en la dinámica económica que replica, a su vez, en la dinámica socio-demográfica y en la estructura político-institucional, produciendo crisis de gobernabilidad de las ciudades".

La segregación socioespacial, se ha convertido en un problema relevante en las ciudades latinoamericanas, especialmente la segregación asociada a los grupos pobres. De acuerdo a lo planteado por Sabatini et al (2001), se vincula a la distancia que deben recorrer para llegar a sus lugares de trabajo. A su vez, los barrios pobres no logran atraer la localización de servicios, oficinas y lugares de trabajo como ocurre con las zonas donde se localizan los grupos de mayor estatus socioeconómico.

La segregación socioespacial es propia de las ciudades y hace referencia a los aspectos negativos a que está asociada por sus causas socioeconómicas, pues a grandes rasgos, actúa como mecanismo de reproducción de las desigualdades socioeconómicas, de las cuales ella misma es una manifestación (Rodríguez y Arriagada, 2004).

La emergencia de los estudios de segregación socioespacial en esta parte del continente, se han intensificado en las últimas décadas ya que los efectos y las nuevas formas que presenta, influyen de manera notable en la configuración socio territorial de las ciudades latinoamericanas.

Es en la ciudad donde la segregación socioespacial se manifiesta de manera significativa, la cual en la última década lo hace por medio de la separación espacial entre las áreas residenciales de grupos de altos ingresos y las áreas residenciales de población en condición de pobreza. Los patrones de ocupación residencial del suelo urbano son resultado de la planificación urbana basada en la separación espacial de actividades. En algunos casos, estos patrones han sido resultado de algunas intervenciones en la ciudad como la renovación urbana que genera un proceso de gentrificación del centro histórico, el cual aleja a los habitantes pobres de este sector, siendo reemplazados por habitantes de altos ingresos. Además, la modernización urbana ha provocado que se construyen nuevas redes viales y grandes centros comerciales que refuerzan la segregación, surgiendo a la par nuevas tipologías de uso residencial como los barrios cerrados los que se encuentran aislados, por grandes murallones vigilados en todo momento, de la población de escasos recursos (Lungo y Baires, 2001).

En relación a lo anterior, se indica que los patrones tradicionales de segregación en las ciudades de América Latina están cambiando debido a la 
proliferación de nuevas comunidades cerradas destinadas a grupos crecientes con ingresos altos y medianos y a la creación de centros comerciales y complejos de oficinas en áreas más modernas fuera de los primeros enclaves urbanos. En São Paulo, Santiago, Buenos Aires y Ciudad de México, por nombrar sólo algunas de las ciudades más grandes y dinámicas, estas construcciones incluso están surgiendo al lado de áreas de bajos ingresos. Al mismo tiempo, estos cambios en los patrones de segregación reducen las distancias físicas entre los grupos socioeconómicos, y permiten el acceso de la población pobre a las instalaciones comerciales “modernas”y a los espacios públicos mejorados (Greenstein et al, 2000).

En el caso particular de Chile, se evidencia un cambio profundo en los patrones de segregación espacial en las grandes ciudades Chilenas (Ortiz \& Escolano, 2013; Sabatini, 2003; Sabatini \& Cáceres, 2004; Sabatini, Cáceres, \& Cerda, 2001b). En efecto, hasta hace unas décadas el patrón de segregación espacial en las ciudades nacionales era bien claro, en estas se configuraban grandes áreas homogéneas en términos socioeconómicos, las que eran fáciles de reconocer a través de un análisis de distrito censal, o aún más en el caso de las áreas metropolitanas incluso a nivel comunal. La teoría indicaba que los estratos bajos se ubicaban preferentemente en la periferia urbana, en lugares mal servidos y mal conectados (Hidalgo, 2007; Hidalgo, Zunino, \&Álvarez, 2007; Lambiri \& Vargas, 2011; Peters, 2009), en tanto que los estratos altos, se localizaban en el otro extremo de la ciudad, alejados de la pobreza y con expedito acceso a las funciones y servicios urbanos. Sin embargo, este panorama ha cambiado lentamente a partir de la globalización económica de nuestro país (Sabatini, 2000), la que ha provocado un proceso relocalización de los estratos medios y altos al interior de la mancha urbana, grupos que se han trasladado sus residencias a sectores periféricos, adyacentes a los espacios que antes ocupaban casi exclusivamente, los estratos más bajos. A diferencia de lo evidenciado por la literatura en otras latitudes, especialmente Norteamérica y Europa, la localización periférica de los estratos medios y altos no ha ido acompañada de un proceso de expulsión de los grupos sociales bajos que habitaban en esos lugares.

Las dinámicas antes descritas han gatillado una disminución de la escala geográfica de la segregación, generando que los estratos bajos y altos comiencen a habitar en condiciones de proximidad espacial, abriendo con ello espacios de interacción social entre grupos socioeconómicamente disímiles (Sabatini \& Salcedo, 2007).

No obstante lo señalado en el párrafo anterior, aun es importante la segregación espacial de la población de escasos recursos. Esta situación, ha motivado por parte del Estado, la promulgación de políticas de vivienda social, la que en su espíritu apuntaba a una integración social y por lo tanto a una disminución de los niveles de segregación, situación que en la práctica no aconteció debido a que a que los valores del suelo en las zonas centrales son prácticamente inalcanzables para los ingresos de la población 
de bajos ingresos y los subsidios aportados por el Estado. Es así que Vergara (2014) sostiene que la política de vivienda social se ha constituido durante las últimas tres décadas, como un mecanismo que contribuye a expulsar a los estratos bajos hacia la periferia urbana, concentrando a este grupo social en espacios monofuncionales y mal servidos, gatillando con ello fenómenos de exclusión social que han dado origen a un nuevo tipo de pobreza urbana, la pobreza de los con techo, verdaderos guetos donde prima la cultura de la desesperanza (Rodríguez \& Sugranyes, 2004).

La investigación sobre la segregación socioespacial en Chile, se ha concentrado principalmente en las ciudades mayores y en menor medida en las intermedias, por lo tanto cabe preguntarse ¿Qué sucede con la segregación socioespacial en las ciudades menores?, ¿la política de vivienda social ha generado mayores niveles de segregación socioespacial en estas ciudades?. Estas preguntas no se han explorado hasta el momento por parte de la literatura, por lo que se vuelve necesario ampliar la mirada hacia las ciudades menores ${ }^{1}$, intentando con ello reconocer si en estas se repiten los fenómenos ya evidenciados para las ciudades mayores o intermedias. En consideración a lo anterior, la investigación tuvo como objetivos analizar la segregación socioespacial, le percepción de los habitantes y autoridades públicas sobre esta y el efecto de la aplicación de las políticas de vivienda sobre la segregación en una ciudad pequeña como Angol, ubicada a 550 Kms al sur de Santiago de Chile.

La estructura de este trabajo es la siguiente: en primer lugar se dará a conocer el área de estudio, posteriormente se abordarán las políticas estatales que guían el desarrollo urbano en Chile al igual que la literatura escrita sobre segregación, sus principales perspectivas teóricas y metodológicas, luego se dará cuenta de la estrategia metodológica empleada para el cumplimiento de los objetivos haciendo hincapié en la integración de la dimensión cuantitativa y cualitativa para abordar el caso de estudio, luego se presentarán los resultados emanados de la aplicación metodológica, para concluir analizando, en base a la teoría existente, los principales resultados

\section{Descripción del área de estudio}

Angol se ubica en la parte nor-oeste de la región de la Araucanía, Chile, cercana al límite de la región del Biobío. Actualmente es la capital de La Provincia de Malleco, y según datos del CENSO 2002, tiene una población total que alcanza los 48.996 habitantes, de los cuales 43.801 viven en el área urbana y 5.195 en zonas rurales, constituyéndose así como la tercera comuna más poblada de la Región, luego del área metropolitana TemucoPadre Las Casas. Su zona urbana está compuesta por un total de 7 sectores o llamados también Distritos Censales, estos son Cañón, Hospital, Regimiento, Centro, Rosario, Estación y Huequén (Figura $N^{\circ} 1$.). 


\section{Figura $N^{0} 1$. Localización de la ciudad de Angol}
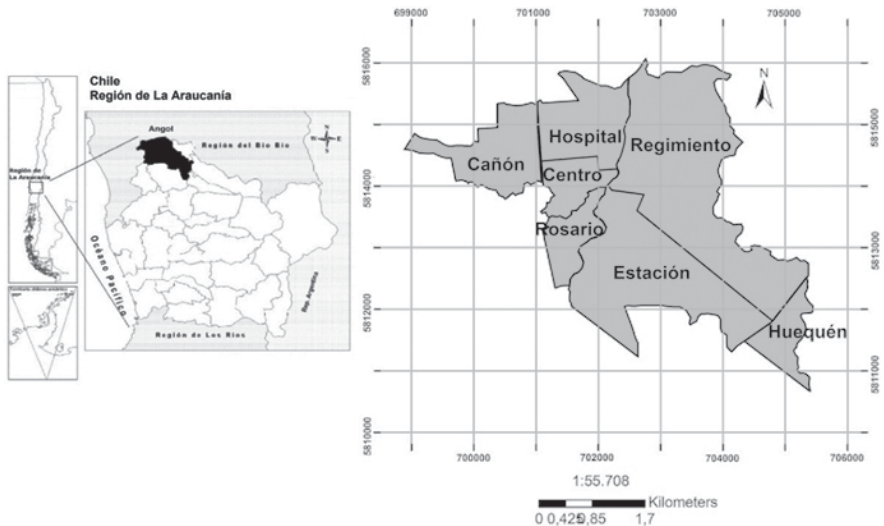

Fuente: Elaboración propia.

Fue fundada en 1553 en plena zona "fronteriza”en el contexto de la guerra de Arauco. Su carácter fronterizo y neurálgico en este largo conflicto, hizo que la ciudad fuera destruida en seis ocasiones, refundándose por última vez en 1862, esta vez en el contexto de la ocupación de la Araucanía. Luego de su última fundación se convirtió en uno de los puntos neurálgicos de la Araucanía, a ella concurrían comerciantes nacionales, colonos recién llegados a la zona y mapuches que venían desde la cordillera de la costa o desde valles de los ríos cercanos para intercambiar sus productos. A inicios del siglo XX la ciudad se transformó en un importante polo de desarrollo económico, ya que en ella confluían diferentes ramales de ferrocarril que transportaban el trigo proveniente principalmente desde la zona de Traiguén, convirtiéndose asíen la puerta de lo que por aquel tiempo era "el granero de Chile", sin embargo, el cierre de los mercados internacionales que se abastecían con el trigo de la Región debido a la baja competitividad del mercado local, provocó que la ciudad entre en un proceso de estancamiento, el que se ve reforzado por la creciente concentración de la actividad comercial y administrativa en la ciudad de Temuco, la que en los inicios del siglo XX pasa a ser la principal ciudad de la Región. La globalización económica y la especialización territorial ha provocado que la ciudad reorientara su economía hacia la actividad forestal, no obstante, los pocos beneficios locales que produce esta actividad, ha generado que los indicadores locales continúen estancados, lo que se ha reflejado en una pérdida de la importancia relativa que esta ciudad tiene sobre la región.

Paradójicamente, la superficie urbana durante las últimas décadas se ha expandido rápidamente. La razones de esta situación se encuentran esencialmente en la acción que el Estado ha desarrollado en la ciudad, a partir de 
la entrega de viviendas sociales. Esto ha provocado que Angol esté experimentando en la actualidad una serie de transformaciones urbanas.

Ante el panorama descrito, cabe preguntarse si es que la ciudad en general continúa replicando aquellas consecuencias del desarrollo urbano que ya han sido evidenciadas en otras ciudades mayores y particularmente sobre la segregación socioespacial local, sobre todo considerando que el crecimiento urbano de Angol se ha sustentado sobre la construcción de viviendas sociales.

\section{Las reformas urbanas neoliberales}

La crisis económica que se experimentó a inicios de la década del setenta, puso en jaque al sistema económico mundial. Con el fin de dar una rápida salida de la crisis se argumentódesde los círculos neoliberales, que tomaban fuerza por aquella época, que la causa de la coyuntura era el gran tamaño que había adquirido el Estado. Consecuentemente con lo anterior se aplicaron en Chile una serie de reformas por los llamados "Chicago Boys", economistas neoliberales, que tuvieron como consecuencia una vertiginosa retirada del Estado, y paralelamente la ampliación del mercado en el rol y consecución del desarrollo nacional.

Las modificaciones implementadas influyeron tanto en las estructuras sociales como espaciales de nuestro país, y particularmente en lo que se refiere a la ciudad al menos dos reformas son esenciales: La nueva política de desarrollo urbano y la nueva lógica que adquiere la vivienda social.

La política de desarrollo urbano promulgada en 1979 liberaliza el suelo urbano, establece que el Estado ya no es quien debe planificar y regular el crecimiento de la ciudad, sino que debe ser el mismo mercado a través de la oferta y la demanda lo que va a regular el uso del suelo y la distribución de este. El desarrollo urbano por consiguiente queda en manos de las empresas constructoras e inmobiliarias (Borsdorf, Sánchez, \& Marchant, 2008), y el suelo se ajusta completamente a la nueva lógica de contracción Estatal.

Por otro lado, la política habitacional de la dictadura militar establece que la vivienda es un derecho que se logra con esfuerzo y ahorro, acción en la cual la familia y el Estado comparten cuotas de responsabilidad, ya que la vivienda no es un regalo del Estado (MINVU, 1976). La fórmula es simple, para acceder a una vivienda la familia en primer lugar debe tener ahorros, y con ello solicitar un subsidio al Estado, si este aporte más el ahorro de la familia no es suficiente para la compra de una vivienda, la familia tiene la opción de solicitar un crédito a alguna entidad bancaria privada (Casgrain, 2010). Se diseñan dos mecanismos de financiamiento de la vivienda social: primero un subsidio habitacional para aquellas familias que puedan tener capacidad de ahorro y acceso al crédito para la vivienda, y segundo un subsidio a la construcción de viviendas para grupos con muy escaza posi- 
bilidad de ahorro y nula posibilidad de crédito (Simian, 2010). Con la llegada de la democracia los lineamientos centrales de estas políticas no sufren modificación alguna, aún más, se perfeccionan e intensifican.

La creciente especulación y presión inmobiliaria por ciertos sectores de la ciudad, ha contribuido a un aumento significativo de los valores de suelo urbano en todas las ciudades nacionales, esto ha limitado las posibilidades los estratos socioeconómicamente más bajos de habitar en los sectores consolidados y mejor servidos, teniendo que necesariamente obtener viviendas particulares o bien sociales en los márgenes de la mancha urbana, contribuyendo asía un aumento en la homogeneidad socioeconómica en los anillos periféricos.

Los efectos de la concentración de las viviendas sociales en las ciudades nacionales han sido estudiadas desde distintas perspectivas, algunos han puesto la mirada sobre la manera en que la vivienda social contribuye históricamente a construir el espacio urbano (Hidalgo, 2005, 2007), otros sobre la contribución de esta en la segregación social y espacial de las ciudades (Garín, Salvo \& Bravo, 2009a), como asimismo en los efectos sociales de la construcción de grandes conjuntos de vivienda como la guetificación (Sabatini \& Brain, 2008), el rompimiento de los lazos sociales (Ducci, 2005), la delincuencia (Garrido \& Astorga, 2013) y el estigma barrial (Cornejo, 2012). Estos estudios han concluido que la política de vivienda social y la de desarrollo urbano han contribuido a aumentar la segregación social y espacial de los estratos más bajos, los que han sido traslados a vivir al borde de la mancha urbana, generando con ello también una serie de problemas de transporte, acceso a servicios, delincuencia, estigmatización territorial, etc.

\section{La segregación socioespacial: enfoques y metodologías}

La segregación es entendida como la "aglomeración geográfica de familias de una misma condición o categoría social, como sea que se defina esta última, social, racialmente o de otra forma” (Sabatini, Cáceres, \& Cerda, 2001: 3). Los primeros estudios referidos a esta temática fueron realizados en el mundo anglosajón hacia inicios del siglo XX, en ellos se abordó la homogeneidad que experimentaban algunos sectores de la ciudad norteamericana donde habitaban exclusivamente inmigrantes que llegaban hasta EE.UU buscando nuevas oportunidades laborales. Estos estudios estaban fuertemente influenciados por la perspectiva darwiniana, de ahíque plantearan que en la ciudad se aplicaba "la ley del más fuerte", es decir los mejores lugares por naturaleza eran siempre ocupados por aquellos estratos sociales de mayor status (Garín, Salvo, \& Bravo, 2009b; Molina, 2001).

Contrario a lo que sucede en el mundo anglosajón, la literatura latinoamericana se ha preocupado de estudiar la segregación que se ha experimentado a nivel de estrato socioeconómico, buscando entender cómo es 
que los distintos grupos socioeconómicos se distribuyen en el espacio urbano y el por quéestos se concentran en ciertos lugares de la ciudad.

Existen dos perspectivas teóricas sobre las cuales se construyen los estudios referidos a la segregación socioespacial, la primera de ellas ofrece una mirada estructuralista (Garín, Salvo, \& Bravo, 2009b), donde se asume que el capitalismo es un sistema desigual por naturaleza y que aquella desigualdad se expresa de manera casi mecánica en la ciudad, provocando que los grupos sociales habiten alejados entre sí, esta tesis se ha denominado como la del "espejo" (Sabatini \& Brain, 2008), ya que el espacio urbano es el reflejo de lo que sucede a nivel de sistema económico. Si bien la mayoría de los estudios realizados sobre segregación se ha afiliado a esta perspectiva, las críticas sobre ella han enfatizado en la limitada posibilidad que tiene para explicar la situación de algunas ciudades socialmente y espacialmente integradas en contextos económicos nacionales altamente desiguales.

La segunda perspectiva, señala que la segregación es un proceso complejo, que no se produce simplemente por reflejo de aquella desigualdad natural de la macro estructura en el espacio, sino que esta estámediada por factores de tipo sociológicos, culturales y ambientales, de ahíque se ponga énfasis en la manera en las relaciones interpersonales generan segregación (Salcedo \& Torres, 2004). Sobre esta base, es que algunos estudios contemporáneos han reflexionado en torno a la necesidad de avanzar en una definición compuesta de la segregación, reconociendo que esta se compone de una dimensión objetiva y otra cuya esencia es de carácter subjetivo. La dimensión objetiva hace referencia a dos elementos principales, primero, la tendencia de algunos grupos a concentrarse en el espacio urbano, lo que se asocia al grado de dispersión o concentración de los distintos grupos sociales al interior de la ciudad y, segundo, el grado de homogeneidad y/o heterogeneidad social de cada una de las áreas de la ciudad (Barrio-sector-distrito censal, etc.). En tanto, la dimensión subjetiva está vinculada a la percepción que posee la población respecto a las formas de segregación objetiva, esto se asocia por un lado a la percepción que los habitantes tienen sobre la ocupación de parte de ellos del espacio, por ejemplo los sentimientos de ser marginalidad (marginales), de "residuo social”, elementos que se constituyen en la base de la desintegración social, y por otro lado, esta dimensión se relaciona con la identidad de los grupos sociales y el nivel de prestigio de ciertas áreas de la ciudad, lo que se vincula con la existencia de estigmas territoriales (Sabatini \& Sierralta, 2006). Se entiende, desde esta perspectiva, que la segregación de una ciudad o barrio está cruzada por estas dos dimensiones las que establecen relaciones que en ningún caso son unidireccionales y proporcionales entre ellas, esto quiere decir que puede existir alto grado de segregación en la dimensión objetiva, pero no necesariamente suceder lo mismo en materia de la dimensión subjetiva, o al revés, por lo que las dimensiones de la segregación de una ciudad no necesariamente deben coincidir, estas dependen fuertemente del contexto socio-histórico, de la escala, de las dinámicas temporales y espaciales de las variables consideradas y de las relaciones sociales cotidianas que se dan en la ciudad en el cual se insertan. 
La literatura existente, que en su mayoría se enmarca dentro de la perspectiva estructuralista, ha empleado preferentemente metodologías de naturaleza cuantitativa para medir la segregación, destacando en estos estudios el índice de disimilitud de Duncan que mide la segregación de un grupo en comparación con otro (Martori \& Hoberg, 2004), y el índice de aislamiento que mide la probabilidad que un individuo comparta la unidad espacial con un individuo de su mismo grupo (Ibid). El problema que presentan las aproximaciones a la segregación basadas exclusivamente en índices, es que establecen vínculos pocos claros en la relación existente entre la estructura social y espacial, infiriendo, y en algunos casos especulando, a partir de ellos, que el aumento en la segregación espacial es causada por una creciente desigualdad en materia de indicadores económicos y laborales, limitante que emerge esencialmente debido a la naturaleza descriptiva que poseen estos y a la poca capacidad para abrir espacios de interpretación analítica fundamentada. En suma, la utilización de indicadores para medir la intensidad de la segregación, si bien son necesarios y muy útiles, sólo entregan una mirada parcial del fenómeno asociada a la dimensión espacial y objetiva de este, no siendo capaces de explicar la dimensión de la segregación asociada a la percepción subjetiva de esta (Sabatini, 2000). La trascendencia de la dimensión social de la segregación radica en que esta es la base sobre la cual se construyen las relaciones cotidianas entre el nosotros y los otros, posibilitando o impidiendo la interacción entre grupos sociales disimiles en términos socioeconómicos o culturales y gatillando dinámicas de integración o desintegración social.

Si bien es cierto que estos procesos han sido, como hemos mencionado, estudiados para las áreas metropolitanas y algunas capitales regionales, no se ha profundizado en la misma manera en las ciudades pequeñas, de ahí que sea relevante conocer la manera en que se expresa la segregación socioespacial en las ciudades menores y si efectivamente la vivienda social continúa el patrón de localización periférica, o bien si estás ha contribuido de manera significativa a generar procesos de exclusión social tal como se ha documentado por la literatura.

\section{Metodología}

Explorar la relación entre las dimensiones objetivas y subjetivas de la segregación permite establecer las vinculaciones e imbricaciones más profundas que se dan entre la estructura espacial y social. Resulta por tanto, necesario insertar así los datos de los indicadores cuantitativos en contextos interpretativos que permitan dar lecturas específicas a la realidad social que se está estudiando, revelando también luces sobre el contexto histórico en el cual se desarrollan los procesos sociales que están acaeciendo. Eso nos lleva a plantear en línea con lo propuesto por Saravi (2008) que la segregación urbana está inserta en procesos sociales macro-estructurales, esto quiere decir que en parte la distribución de los grupos al interior del espacio urbano es resultado de procesos sociales de des- 
igualdad y exclusión que se objetiva, aunque no de manera mecánica, en el espacio urbano. Pero a la vez, la estructura espacial retroalimenta los procesos de la estructura social, por ejemplo limitando el acceso a oportunidades y segmentando la ciudad, abriendo procesos de deterioro del vínculo social, la sociabilidad y por tanto asentando los procesos de desigualdad y exclusión social.

Con el fin de explorar tanto la dimensión espacial como la social de la segregación, se optó por la utilización de una metodología mixta, donde se integren técnicas cuantitativas y cualitativas. Si bien es cierto que la literatura ha utilizado mayormente la perspectiva cuantitativa, consideramos que pensar exclusivamente en estos términos la segregación conlleva una serie de limitantes, ya que la utilización de indicadores impiden escudriñar e interpretar en profundidad los problemas existentes en la relación entre la estructura espacial y social y por tanto entre las dimensiones objetivas y subjetivas de la segregación (Saraví, 2008). Lo anterior establece la necesidad de que se produzca una integración metodológica que permitan no solo mirar la dimensiones espaciales de la segregación sino que también la percepción que poseen los habitantes de la ciudad respecto a estos fenómenos y la manera en que les impactan (Flores, 2003). Sin embargo, hay que hacer algunas advertencias respecto a esta integración, primero las dos dimensiones de la segregación no necesariamente deben coincidir, así por un lado los índices pueden mostrar mayor segregación pero no necesariamente esto se debe traducir forzadamente en una percepción más maligna de esta, ahora bien, el hecho de que puedan funcionar con dinámicas disimiles, no necesariamente estas son independientes de la otra, y esto nos lleva a la segunda advertencia, hay que tener cuidado con forzar la relación entre la estructura espacial y social, la segregación por tanto debe pensarse en términos de relaciones complejas y múltiples, ya que el fenómeno puede asumir modalidades diferentes que dependen, como expresamos, de las particularidades del caso de estudio.

Metodológicamente, para los aspectos cuantitativos, se utilizó información proveniente de los censos del año 1992 y 2002 a fin de calcular la variaciónn del índice de disimilitud de Duncan (la información del censo 2012 no se encuentra disponible) considerando espacialmente el distrito censal con la variable del jefe de hogar con más de 12 años de escolaridad ${ }^{2}$. Se empleó esta variable como una aproximación al nivel socioeconómico del hogar (Molinatti, 2013). El índice de disimilitud varía entre 0 y 1, el 0 implica la ausencia de segregación, en tanto que el 1 implica una segregación máxima. Para analizar los resultados de este indicador, se tomó la categorización empleada por Briggs (2001), quien establece tres niveles de segregación: severa $(0,6-0,9)$; moderada $(0.3-0,6)$; Baja $(0-0,3)$. Finalmente también se optó por realizar una exploración más específica de la distribución de la escolaridad dentro del radio urbano, por ello es que se procedióa obtener el promedio de escolaridad de los jefes de hogar por manzana para el año 2002. 


\section{Figura $N^{\circ} 2$. Cálculo de índice de disimilitud de Duncan.}

$$
D=\frac{1}{2} \sum_{i=1}^{n} \mid \begin{aligned}
& \frac{x_{i}}{X}-\frac{y_{i}}{Y} \mid \\
& \text { Yi: población del grupo mayoritario en cada distrito censal } \\
& \text { Y: Total de población de este grupo en el municipio. }
\end{aligned}
$$

Fuente: Elaboración propia

La dimensión cuantitativa también fue útil para reconocer el impacto que ha tenido la construcción de viviendas sociales sobre la segregación espacial en la ciudad de Angol, para ello se obtuvo información desde los registros de permisos de edificación de la dirección de obras municipales, en ellos se recolectó información referida a los conjuntos de vivienda social que fueron construidos dentro del radio urbano de Angol entre 1992 y 2012. Con esta información se elaboró una base de datos de los conjuntos de vivienda los que posteriormente fueron localizados y delimitados con trabajo en terreno. Estos datos permitieron reconocer las tendencias espaciales que ha tenido la construcción de los conjunto de vivienda social en la ciudad, reconociendo si se encuentran o no concentradas en algún lugar específico de la ciudad aproximándonos con ello a la segregación espacial local. Además se emplearon estos datos para calcular la superficie construida de vivienda social entre los años que involucra este estudio, para ello se elaboró cartografía que fue analizada con el software Arcview 10.

Por otro lado, la dimensión cualitativa del estudio se empleó con el fin de conocer los problemas de exclusión social derivados de la localización de la vivienda social en la ciudad. Para ello se aplicó una entrevista semi-estructurada, durante los meses de septiembre y octubre de 2013, a ocho personas, cuatro de ellas residentes de diferentes conjuntos de vivienda social que se ubican en la ciudad y otras cuatro a personas encargadas de la toma de decisiones técnicas y políticas a la hora de construir los conjuntos de vivienda social, a este grupo lo llamaremos tecnopolitas (Zunino, 2006). Las entrevistas fueron grabadas previa autorización de los participantes, los que permanecerán en anonimato.

En suma, el empleo de una estrategia mixta permite obtener una idea más acabada de la segregación, explorando tanto las dimensiones objetivas y subjetivas de este fenómeno. Las metodologías cuantitativas y en este caso el índice de Duncan, pueden ser útiles para entregar una magnitud del problema y la dimensión de la concentración y/o distribución espacial de los grupos sociales al interior de la ciudad, sin embargo, no rescatan el elemento subjetivo que elaboran las personas y la forma de ocupar el espacio, esto puede ser complementado por el 
empleo de una técnica de carácter cualitativo como lo es la entrevista, que permite profundizar en el estudio de la segregación subjetiva en un contexto particular.

\section{La vivienda social y su localización en la estructura urbana de Angol}

Según datos del MINVU (2007) desde 1993 a 2003 la superficie urbana de Angol se amplió de forma significativa, pasando desde las 391,54 a 703,36 hectáreas, logrando con ello en tan sólo en diez años, un aumento en la superficie ocupada de un $86,54 \%$. El potente crecimiento urbano se ha frenado en el nuevo milenio, pues en 2012 la superficie urbana de la ciudad alcanzaba las 866 hectáreas (Vergara \& Rozas, 2013). Ahora bien, la interrogante que nos compete explorar en este trabajo, es cuánto de este crecimiento se explica a partir de la acción del Estado reflejada en la construcción de la vivienda social. Según los datos recolectados en el registro de edificación de la municipalidad de Angol desde 1992 a 2012 se han construido dentro del radio urbano un total de 107 hectáreas de vivienda social, es decir, cerca de un 20\% del crecimiento que la ciudad ha experimentado durante las últimas décadas, se explica por los nuevos conjuntos de vivienda que se han construido, viviendas que en promedio alcanzan los $32,3 \mathrm{~m} 2$.

La localización de la vivienda social dentro del periodo de estudio, ha estado concentrada en ciertos sectores de la ciudad. Como se puede apreciar en la tabla 2, dos distritos censales se constituyen como los principales receptores: el distrito Estación y el distrito Regimiento.

El Distrito Estación ha acogido el 63,59\% de la vivienda social construida durante el periodo de este estudio, las razones se deben esencialmente a que hacia el año 1992 este era el distrito censal que tenía más espacio libre para sostener el crecimiento urbano local, además de que el valor del suelo en él era más bajo. A medida que se ha ido construyendo, el espacio libre que existía en este distrito ha disminuido de manera considerable, situación que ha obligado a los agentes urbanos locales a localizar los nuevos conjuntos de vivienda social en otros distritos censales, este ha sido particularmente el distrito Regimiento que en los últimos años se ha encargado de recibir gran parte la vivienda social construida en la ciudad. También destaca como receptor el distrito Hospital, sobre todo en la década del noventa e inicios del nuevo milenio. 
Polis, Revista Latinoamericana, Volumen 15, No 44, 2016

Tabla $\mathbf{N}^{\circ} 1$. Viviendas sociales construidas por distrito censal en Angol, 1992-2012.

\begin{tabular}{|c|c|c|c|c|c|c|c|c|}
\hline Año & Total & Cañón & Centro & Hospital & Rosario & Regimiento & Estación & Huequén \\
\hline 1992 & 293 & - & - & 8 & - & - & 285 & - \\
\hline 1993 & 339 & - & - & 53 & - & 234 & 52 & - \\
\hline 1994 & 118 & - & - & 28 & - & - & 90 & - \\
\hline 1995 & 275 & - & - & - & - & 35 & 240 & - \\
\hline 1996 & 125 & - & - & - & 32 & - & 93 & - \\
\hline 1997 & 199 & - & - & 16 & - & 61 & 122 & - \\
\hline 1998 & 150 & - & - & - & - & - & 150 & - \\
\hline 1999 & 200 & - & - & - & - & - & 200 & - \\
\hline 2000 & 145 & - & - & - & - & - & 145 & - \\
\hline 2001 & 349 & - & - & - & - & 109 & 240 & - \\
\hline 2002 & 78 & - & - & - & - & 78 & - & - \\
\hline 2003 & 180 & - & - & 36 & - & - & 144 & - \\
\hline 2004 & 261 & - & - & 76 & - & - & 185 & - \\
\hline 2005 & 133 & - & - & 90 & - & - & 43 & - \\
\hline 2006 & 239 & - & - & - & - & - & 140 & 99 \\
\hline 2007 & 166 & - & - & - & - & 105 & 61 & - \\
\hline 2008 & 347 & - & - & - & - & 264 & 83 & - \\
\hline 2009 & 0 & - & - & - & - & - & - & - \\
\hline 2010 & 40 & - & - & - & - & - & 40 & - \\
\hline 2011 & 0 & - & - & - & - & - & - & - \\
\hline 2012 & 0 & - & - & - & - & - & - & - \\
\hline Total & 3637 & $\mathbf{0}$ & $\mathbf{0}$ & $\mathbf{3 0 7}$ & $\mathbf{3 2}$ & $\mathbf{8 8 6}$ & $\mathbf{2 3 1 3}$ & $\mathbf{9 9}$ \\
\hline & & & & & & & & \\
\hline 1969 & - & - & - \\
\hline
\end{tabular}

Fuente: Elaboración propia en base a datos de la Dirección de obras municipales, Angol. 


\section{Figura $\mathrm{N}^{\circ} 3$. Conjuntos de vivienda social construidos en Angol, 1992-2012.}

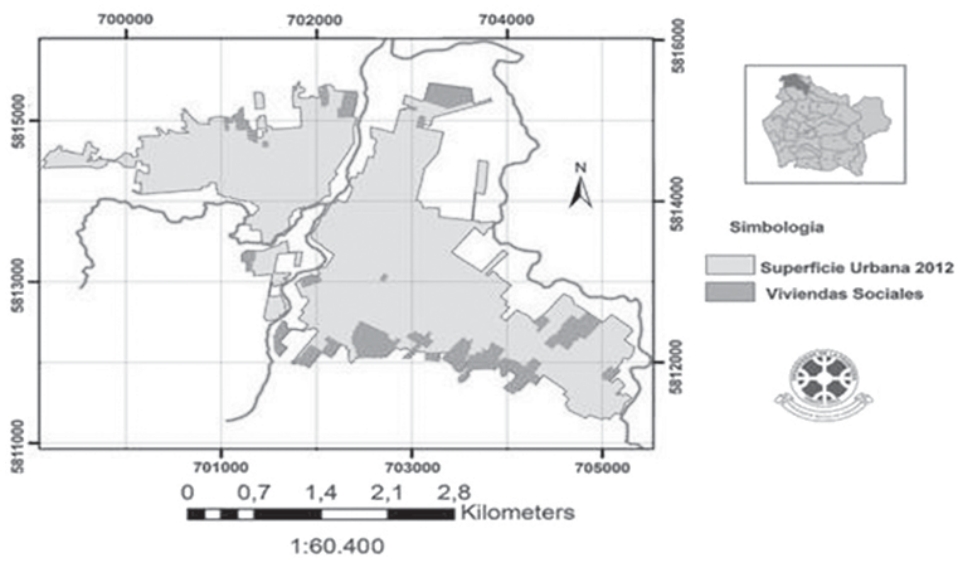

Fuente: Elaboración propia en base a datos de la Dirección de obras municipales, Angol.

La vivienda social no se ha construido de manera dispersa dentro de los distritos censales mencionados, por el contrario, se ha tendido a concentrar en lugares acotados de estos (Figura 3.). El caso más representativo de aquello es el eje Bunster, una arteria vial estructurarte que recorre la parte sur de la ciudad y que ha recibido la construcción de la mayoría de los conjuntos de vivienda social en el distrito Estación. Para el caso del distrito censal Regimiento la construcción se concentra en Los Confines Norte y en el caso de Hospital en Villa Galvarino y Nahuelbuta.

Por el contrario, hay distritos que prácticamente no ha recibido vivienda social en el periodo de este estudio, es lo que sucede con los distritos Centro y Cañón. En el primero de ellos el valor del terreno es excesivamente alto para los parámetros considerados en los proyectos de vivienda social, en tanto que en el segundo la razón se debe más bien a la falta de espacio libre para la construcción y la topografía irregular que lo rodea, ya que se encuentra a los pies de la cordillera de Nahuelbuta.

Según datos recolectados en las entrevistas aplicadas a los tecnopolitas, las razones esenciales a la hora de decidir la localización de un conjunto de vivienda social son: el valor del terreno; el espacio disponible al interior de los distritos censales; y las condiciones topográficas del terreno, si por ejemplo es inundable. Luego del terre- 
moto del año 2010, se ha incorporado también como factor en la localización el riesgo sísmico que presenta el sector donde se pretende construir.

Así, la localización de la vivienda social dentro del radio urbano de Angol ha estado supeditada, al igual que en todas las ciudades nacionales, a la rentabilidad, criterios preferentemente económicos que regulan el desarrollo urbano nacional. Ante esto la municipalidad local ha intentado establecer algunas regulaciones, sin embargo, estas no han sido eficaces en evitar la concentración espacial de la vivienda social, contribuyendo asía configurar dentro de la mancha urbana ciertos espacios homogéneos en términos socioeconómicos, y por consiguiente contribuyendo a aumentar la segregación espacial en sectores específicos de la ciudad.

\section{Vivienda social y segregación espacial en la ciudad de Angol}

En el acápite anterior, dimos cuenta que la vivienda social en Angol, se encuentra localizada en ciertos sectores específicos de la mancha urbana, sin embargo, cabe preguntarse si este fenómeno ha contribuido a aumentar los niveles de segregación espacial en la ciudad, para ello exploraremos la evolución de la segregación espacial a nivel de distrito censal, medida a través del índice de disimilitud de Duncan.

En términos generales, los niveles de segregación espacial de cada uno de los distritos censales de Angol se mantienen relativamente estables entre 1992 y 2002. Tal como se aprecia en la tabla 1. el distrito que más ha variado es el distrito Rosario $(-0,051)$ el que pasó de poseer una segregación severa a moderada, le siguen el distrito Hospital (0,014), el distrito Cañón $(-0,0096)$, y finalmente el distrito Estación $(0,0003)$, todos con segregación moderada para ambos años de estudio. Los distritos Huequén y Regimiento no modifican sus niveles de segregación.

Pasemos a revisar la situación específica de cada distrito censal, para explicar de esa manera la influencia que tiene la vivienda social y otros fenómenos locales en los resultados obtenidos. 
Tabla №2. Evolución de la segregación espacial en Angol, 1992-2002

\begin{tabular}{|l|l|l|l|l|l|c|c|}
\hline & $\begin{array}{c}\text { Duncan } \\
\mathbf{1 9 9 2}\end{array}$ & Nivel & $\begin{array}{c}\text { Duncan } \\
\mathbf{2 0 0 2}\end{array}$ & Nivel & Evolución & $\begin{array}{c}\text { \% Población } \\
\text { con +12 años } \\
\mathbf{1 9 9 2}\end{array}$ & $\begin{array}{c}\text { \% Población } \\
\text { con +12 años } \\
\mathbf{2 0 0 2}\end{array}$ \\
\hline Centro & No aplica & & $\begin{array}{c}\text { No } \\
\text { Aplica }\end{array}$ & No aplica & No aplica & 24,5 & 37,6 \\
\hline Hospital & 0,516 & Moderada & 0,502 & Moderada & Mantiene & 15,1 & 20,9 \\
\hline Cañón & 0,510 & Moderada & 0,500 & Moderada & Mantiene & 2,8 & 6,2 \\
\hline El rosario & 0,617 & Severa & 0,565 & Moderada & Baja & 16,1 & 35,1 \\
\hline Huequén & 0,500 & Moderada & 0,500 & Moderada & Mantiene & 3,2 & 4,9 \\
\hline Estación & 0,501 & Moderada & 0,501 & Moderada & Mantiene & 25,6 & 40,9 \\
\hline Regimiento & 0,500 & Moderada & 0,500 & Moderada & Mantiene & 5,8 & 13,4 \\
\hline
\end{tabular}

Fuente: Elaboración propia.

El distrito Rosario poseía en 1992 un total de 201 viviendas, para el año 2002 la cifra se elevó a 518. Este crecimiento en el número de viviendas se explica, en parte, por la construcción de algunos conjuntos de vivienda social en el sector (32 viviendas), sin embargo, este fenómeno ha ido acompañado de la construcción de algunas viviendas dirigidas también a estratos medios emergentes, e incluso un barrio privado. Así la construcción de viviendas dirigidas a distintos estratos socioeconómicos contribuyó a disminuir los niveles de segregación espacial, diversificando la base social del sector.

El distrito Hospital y Cañón se mantienen estables en términos de la evolución de su segregación, el primero de ellos acogió durante una parte de la década del noventa algunos grandes conjuntos de vivienda social (Población Nahuelbuta), sin embargo, este proceso fue acompañado de la consolidación de un sector de este distrito (cercano al centro comercial de la ciudad) donde se localizaron preferentemente jefes de hogar con estudios universitarios, esta situación contribuyó a disminuir levemente los niveles de segregación espacial. En tanto, el distrito Cañón durante el periodo de estudio experimentó una expansión de viviendas hacia la ruta que une la ciudad con el parque nacional Nahuelbuta, sin embargo, ese creci- 
miento no fue producto de la vivienda social, sino que más bien viviendas de autoconstrucción, principalmente asociadas a migrantes con bajos niveles de escolaridad provenientes de los sectores rurales de la Cordillera de La Costa, quienes vieron mermadas sus fuentes de trabajo producto de la tecnificación agrícola y el potente avance del suelo forestal, fruto de aquello y a diferencia de otros distritos, el cañón no ha diversificado mayormente su base social durante el periodo de estudio.

El distrito Estación fue el gran receptor de la vivienda social durante el periodo de estudio, esto debería haber contribuido a aumentar los niveles de segregación espacial, sin embargo, también se localizaron en él una serie de iniciativas comerciales e inmobiliarias que atrajeron a la clase media emergente y profesionales universitarios que se instalaron en la ciudad, esta situación contribuyó a que el índice de disimilitud no experimentara un alza significativa en el periodo de estudio, manteniendo sus niveles estables en el tiempo.

El crecimiento habitacional del distrito Huequén durante el periodo de estudio se estancó, manteniendo su base socioeconómica de extracción popular, con bajos niveles de escolaridad, de ahí que no experimente modificación alguna en sus niveles de segregación espacial. La construcción de algunos conjuntos de vivienda social en el sector durante los últimos años (2009-hoy), probablemente contribuirá en el tiempo a aumentar los niveles de segregación espacial.

Finalmente, si bien es cierto que el distrito Regimiento ha recibido una parte importante de la vivienda social en la ciudad, este también ha sustentado su crecimiento espacial en la consolidación de un incipiente mercado inmobiliario a través de la construcción de algunas viviendas dirigidas a clase media alta y alta, promoviendo la localización de habitantes con mayores niveles de escolaridad. Esta dinámica, le ha permitido a este distrito constituirse como el que más integrado socio espacialmente en la ciudad.

Ahora bien, el mantenimiento de los niveles de segregación no implica que todos los distritos presenten los mismos porcentajes de Jefes de Hogar con 12 años o más de escolaridad, para el caso del año 2002 el distrito censal que más porcentaje de jefes de hogar con 12 o más de escolaridad es el Centro (37,6\%), razón que se explica porque en este sector aún persiste fuertemente el hecho de que los locales comerciales se encuentran en la misma propiedad del dueño, el que además habita en alguna construcción dentro del mismo terreno. Le continúa el Rosario (33,4\%), sector que ha experimentado un fuerte crecimiento poblacional en el periodo de estudio ubicándose en este sector habitantes cuyos niveles de escolaridad han sido mayores a los existentes en 1992. El distrito Hospital (20,8\%), muy cercano al centro urbano, mantiene altos niveles de escolaridad para el contexto comunal, ubicándose en este, profesionales asociados al área de la salud. En el Regimiento (19,6\%) sucede algo similar con los profesionales del área de las fuerzas armadas. Finalmente los que presentan los menores 
niveles de escolaridad son el distrito Estación (15,4\%), Cañón (6,3\%) y Huequén (5,5\%), todos ellos conformados históricamente por población de extracción popular que migró desde sectores rurales o ciudades aledañas hasta la ciudad de Angol, fundamentalmente durante la segunda mitad del siglo XX.

Consideramos que la pequeña extensión urbana de la ciudad de Angol, influye en el resultado obtenido por el índice de disimilitud de Duncan, al respecto, los distritos censales no presentan niveles de segregación socioespacial severos, esto producto de que en ellos es posible encontrar población de todos los estratos socioeconómicos habitando en condiciones de relativa proximidad espacial. Sin embargo, el índice de disimilitud esconde la especificidad propia de una ciudad pequeña. De la imagen 2, se puede inferir que hay ciertos lugares específicos dentro de cada distrito censal que concentran niveles similares de escolaridad de los jefes de hogar, la segregación adquiere asíuna magnitud menor en términos espaciales, ya que igualmente dentro de los distritos censales se configuran ciertas áreas homogéneas en términos socioeconómicos. Así podemos reconocer que la vivienda social si ha contribuido a aumentar los niveles de segregación espacial en la ciudad de Angol, sin embargo la manera en que se manifiesta esta es a menor escala, muy diferente a la evidenciada en las grandes ciudades. Resta aún por reconocer si efectivamente la concentración de la vivienda social, en lugares acotados de la mancha urbana, ha gatillados procesos de exclusión social como lo ha evidenciado la literatura en las grandes ciudades nacionales.

\section{Figura $\mathrm{N}^{\circ}$ 4. Escolaridad del Jefe de Hogar por Manzana en la ciudad de Angol. 2002.}

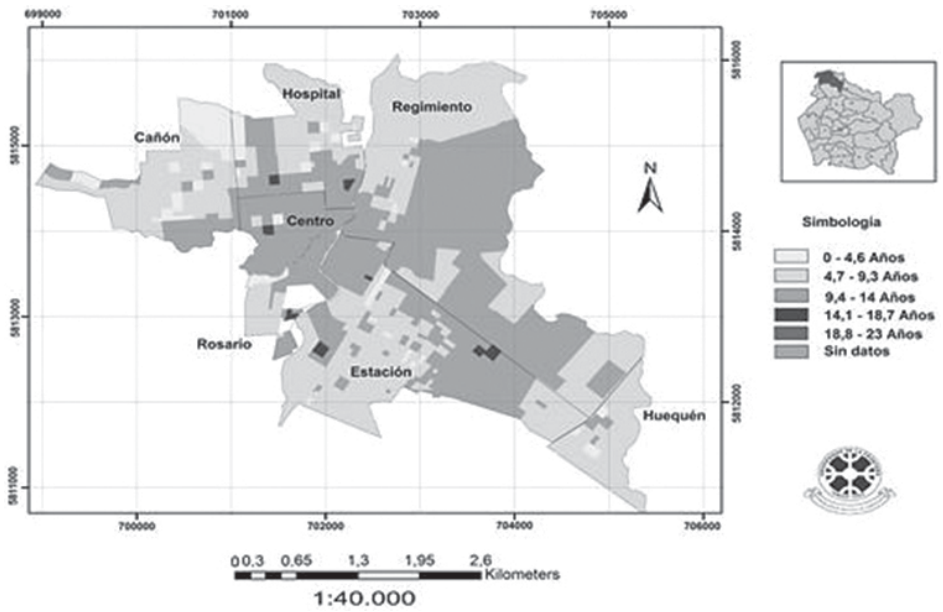

Fuente: Elaboración propia. 


\section{La percepción de la segregación socioespacial en una ciudad pequeña y los "problemas”asociados}

En este acápite se intentará avanzar en la exploración de aquella dimensión subjetiva de la segregación, particularmente para ello concentraremos nuestro análisis en la percepción que los diferentes actores locales y habitantes de vivienda social poseen sobre la segregación a nivel urbano y algunos de los inconvenientes que potencialmente pudieran derivar de este problema social (acceso a servicios, locomoción y estigma territorial).

De la entrevista aplicada, el 100\% de las personas percibe a Angol como una ciudad segregada. Sin embargo, un $75 \%$ de la muestra entiende este problema social como un fenómeno "normal" que es posible encontrar en gran parte de los espacios urbanos nacionales:

"Angol no es distinta de la mayoría de las ciudades de nuestro país, que efectivamente tienen barrios distintos, que suelen estar en los márgenes de la ciudad. Es una ciudad que tiene barrios marginales y tiene sectores distintos, sectores más acomodados...donde se juntan los sectores más acomodados, pero eso sucede en todas las ciudades del país" (Tecnopolita).

En una mirada más amplia, los entrevistados entienden que uno de los rasgos constitutivos de la sociedad Chilena es la predisposición positiva a relacionarse con personas semejantes en términos de la condición socioeconómica y cultural, fenómeno del cual, por cierto, Angol no está ajeno. Ahora bien, perciben que las relaciones sociales también se encuentran limitadas y/o condicionadas por las relaciones de proximidad entre vecinos, así la estructura espacial y distribución de la población de una ciudad por lo tanto influye directamente sobre las posibilidades de contacto entre las personas y consecuentemente en la segregación social.

"Espacialmente yo creo que ocurre lo que ocurre en cualquier ciudad, ponte tú los niños los tienes en un colegio más caro, obviamente los niños se van a relacionar entre ellos, las juntas de vecinos se relaciona entre ellos, pero yo considero que Angol es igual a cualquier otra ciudad donde se relacionan vecinos con vecinos, si hay otra población al lado a lo mejor no lo van a tomar en cuenta, no se van a mirar, a no ser que ellos tengan un tema en común, pero no lo veo como segregación, es como un tema humano que se puede dar en cualquier lado, yo me relaciono con los míos y tú con los tuyos” (Tecnopolita).

Como se indicó, los actores sociales no identifican a la segregación como un problema social, más bien lo entienden como un proceso natural, inherente a la sociedad y toda ciudad nacional. La "naturalización" de la segregación se traduce, por ejemplo, en una carencia de políticas y estrategias locales que enfrenten este tópico como un elemento perjudicial en la calidad de vida de los habitantes de la ciudad. El origen de esta percepción 
bastante particular sobre el fenómeno, radica en que tanto los habitantes locales como los tecnopolitas coinciden en que la segregación en Angol posee una naturaleza muy diferente a la de las grandes ciudades. En efecto, los entrevistados señalan:

"Tú puedes ver condominios de mejor calidad en distintos puntos, puedes ver viviendas sociales en distintos puntos, pero no responde a lo que pasa en Santiago" (Tecnopolita)

"Angol se está expandiendo para donde se puede, indistintamente de las clases sociales (...) pero dentro de los espacios que quedan se van haciendo los comité de vivienda o los condominios, es en cualquier lado, es donde ellos encuentren un lugar. Por ejemplo Villa Betel, están al lado de un supermercado y al lado de la Avenida principal de Angol, entonces no pasa lo que pasa en las ciudades grandes" (Tecnopolita)"

"Al menos en mi Villa está mezclada (...) es un lugar de integración nos conocemos todos y estamos unidos" (Habitante de vivienda social).

Como se indicó en los acápites anteriores, al tratarse de una ciudad pequeña la segregación en Angol adquiere una naturaleza espacial diferente, en donde las distintas clases sociales tienden a habitar en condiciones de relativa proximidad espacial. Se configura así una situación en la cual los barrios de la ciudad poseen habitando en ellos a distintos grupos sociales. La mixtura socio-económica efectivamente es percibida por los entrevistados, situación que incide respecto a la percepción que ellos tienen sobre la segregación más como un proceso "natural” y no tanto como problema social.

La explicación que entregan los actores locales sobre la naturaleza espacialmente reducida de la segregación en Angol, guarda relación con la poca consolidación que han tenido en la ciudad actividades económicas generadoras de grandes riquezas, eso ha limitado la expansión de sectores de altos ingresos y consecuentemente la penetración de la industria inmobiliaria de alto estándar, lo que también explica en buena medida la expansión urbana sustentada en la acción estatal a través de la vivienda social.

“...lo que pasa es que acá en Angol a diferencia de Temuco, Angol es una ciudad de poco recursos y túno vas a encontrar gente ultra millonaria (...) la gente básicamente se dedica a la agricultura o servicios y esos no son rubros donde te enriquezcas, pero no hay gente millonaria" (Tecnopolita)

Sin embargo, la proximidad espacial con la que habitan las distintas clases sociales en la ciudad, no necesariamente se traduce en una menor segregación social y mayor relación pluriclasista.

"Hay microambientes acá en la comuna, que la gente se relaciona en esos microambientes, que además son súper marcados, por ejemplo el am- 
biente militar, todos los militares viven cerca del regimiento, tienen sus propias casas y se relacionan entre ellos, los militares se juntan entre ellos. Es un ambiente cerrado que no es fácil de romper, como también ocurre en los trabajadores de la celulosa, que también ellos tienen su propia Villa y ahílas casas son del porte de la importancia de tu cargo, si eres jefe de áreas tienes la casa más grande, los profesionales tienen una casa más chica, y así, los operarios tienen su propia Villa, distinta, que es la Villa Cordillera. Entonces si hay ambientes distintos, hay efectivamente microambientes..." (Tecnopolita).

En términos sociales, lo que existe en una ciudad pequeña como Angol son "microambientes"caracterizados por alta homogeneidad laboral, socioeconómica y poca permeabilidad. Estos resultados permiten corroborar la idea de que las dimensiones objetiva y subjetiva de la segregación no necesariamente actúan de manera idéntica, ya que si bien espacialmente la ciudad no se encuentra altamente segregada y los grupos socioeconómicos disimiles habitan en proximidad, eso no necesariamente garantiza la existencia de relaciones sociales de carácter pluriclasistas.

Cabe aún preguntar si este contexto urbano local marcado por la segregación espacial a micro escala, ha sido perjudicial para los habitantes de la ciudad gatillando algunos procesos de exclusión funcional como el acceso a los servicio, locomoción, y social como lo es el estigma territorial.

En lo que respecta a la locomoción, la ciudad experimenta una situación particular, existe transporte público de buses urbanos, los que tienen un recorrido fijo que no cubre el total de la ciudad, pero que si conecta la mayoría de los conjuntos de vivienda social con el centro de la ciudad, el problema se presenta en la cantidad de microbuses existentes los que no superan las 15 unidades, de manera que el lapso de tiempo entre uno y otro es muy largo, esto lleva a que la mayor parte de la población recurra al transporte colectivo que es más expedito.

Contrario a lo que sucede con los microbuses urbanos el transporte taxi-auto no tiene recorridos fijos, de manera que depende del chofer el querer transportar a una persona hasta cierto lugar. Esta situación genera ciertos inconvenientes a la hora de ir a algunos sectores periféricos de la ciudad como por ejemplo el Rosario o Población Nahuelbuta, ya que los colectiveros prefieren transitar por las principales vías urbanas.

"En ese sentido hay que tener claro la locomoción colectiva en Angol es muy particular, tenemos una empresa que hace los recorridos dentro de la ciudad, hay una empresa de micros, y están todas las líneas de colectivos, son demasiados colectivos pero ninguno tiene restricción en el recorrido, entonces que es lo que pasa, que el chofer del colectivo te va a llevar a donde el al final más le acomoda" (Tecnopolita). social:

Lo anterior lo reafirma una habitante de un conjunto de vivienda 
"Claro es que los colectivos llegan hasta un cierto punto y después no entran a la villa, los colectiveros dicen que no está dentro de su recorrido, o le cobran dos pasajes para entrar, pero la mayoría no quiere entrar" (Habitante de Vivienda Social).

Por otro lado, según los datos recolectados no existen grandes problemas en torno al acceso a servicios, ya que al ser una ciudad pequeña se reducen considerablemente los tiempos de traslado. Esto se complementa con la dispersión espacial de funciones y servicios como supermercados, farmacias, bancos, estaciones de servicio, etc. a lo largo de la Avenida O’Higgins, principal vía urbana de Angol. Esta situación ha contribuido a que la mayoría de las personas que habitan en conjuntos de vivienda social puedan acceder incluso a pie y en un tiempo que no excede los 30 minutos, a cualquier servicio que necesiten dentro del radio urbano. Consultada respecto a si el vivir en un conjunto de vivienda social ubicado en la periferia urbana le genera problemas a la hora de acceder a servicios, una dueña de casa respondió lo siguiente:

"No, no tenemos problemas, tenemos supermercados, la Copec al frente, el consultorio de la Alemania al lado, no tenemos ningún problema.” (Habitante de Vivienda Social).

Finalmente, los entrevistados concuerdan en que hoy no existen en la ciudad barrios estigmatizados, aquellos que antes se conocían como peligrosos, entre los que cuenta el sector El retiro (autoconstrucción), La cruz del calvario (autoconstrucción), La población Trizano (autoconstrucción), Los Conquistadores (viviendas sociales) o Población Nahuelbuta (viviendas sociales), lentamente comenzaron a cambiar su imagen debido, primero, a que la delincuencia se ha ido erradicando, y segundo, a las políticas de mejoramiento fundamentalmente de infraestructura, como áreas verdes y canchas de fútbol, que se han realizado en estos sectores.

"Hay algunos barrios que nosotros sabemos que tenían algunos problemas, más de delincuencia sí, no sé si estigmatizados. La población Nahuelbuta, la población cumbres, son poblaciones que tuvieron muchos problemas, pero hoy están todas tranquilas, la Trizano era famosa también, pero como que eso ya bajo mucho, porque hoy la Trizano ya es bien céntrica no es periférica. No hay barrios tan complejos acá, porque la mayor parte de los barrios son barrios pobres. (...) además la droga aquí no tiene un arraigo fuerte, aquí hay pobreza pura, no mezclada con la delincuencia ni droga. (...) es pobreza sola, de falta de oportunidades”. (Tecnopolita)

Así se configura por parte de los diferentes actores sociales de la ciudad, la percepción de que la segregación socioespacial a micro escala que evidencia Angol no es perjudicial en materia de acceso a servicios, transporte urbano ni estigma territorial. Esta evidencia se constituye también en una de las bases para entender el desconocimiento que los actores locales tienen de la segregación como un problema social. 


\section{Reflexiones finales: la segregación socioespacial de ciudades pequeñas durante la era neoliberal}

La segregación no solo se manifiesta en términos espaciales, sino que tambiénn en términos sociales, las dos dimensiones están interrelacionadas. En el caso de Angol, la segregación espacial se ha mantenido relativamente estable en todos los distritos censales urbanos, si bien es cierto que hay variaciones leves demostradas con la aplicación del índice de Duncan, estás no han sido capaces de trasformar profundamente la distribución espacial de los grupos socioeconómicos al interior de la ciudad entre 1992 y 2012. Se puede plantear entonces que la expansión y consolidación del capitalismo neoliberal no ha contribuido a modificar sustancialmente la composición social del espacio en esta ciudad pequeña. En buena medida, esto puede ser entendido en un contexto urbano como el de Angol, en el cual el capital inmobiliario no ha penetrado con la misma intensidad de las grandes ciudades.

Lo anterior es evidente cuando se acota la mirada a lo que sucede al interior de los distritos censales, porciones urbanas relativamente pequeñas que albergan, en el caso de Angol, población de todos los estratos socio-económicos. Así, el tejido socio-espacial de esta ciudad pequeña se caracteriza por un alto nivel de mixtura social, en la cual grupos disímiles en términos de su condición socio-económica habitan en condiciones de proximidad espacial. La segregación a una escala espacial reducida parece formar parte de la naturaleza propia de las ciudades pequeñas, y se constituye en una condición histórica, que en este caso, no ha experimentado grandes modificaciones a la luz de las transformaciones de las últimas décadas.

A diferencia de lo que sucede en las grandes ciudades, Angol no ha avanzado hacia la configuración de una sociedad dual de estructura socioeconómica polarizada, la reestructuración capitalista de la década del ochenta dio como resultado la consolidación de actividades económicas de baja rentabilidad y por tanto no se ha traducido en un aumento notorio de estratos de altos ingresos, situación que puede ser observada en la baja cualificación que en general posee la población local y en la casi nula construcción de conjuntos residenciales de alto estándar en la ciudad (Ver Vergara, 2015).

La expansión urbana de Angol en el periodo de capitalismo neoliberal se ha sustentado esencialmente en la construcción de conjuntos de vivienda social por parte del Estado, los que si bien han ayudado a concentrar a los estratos socioeconómicos bajos en la periferia urbana y en algunos sectores específicos de los distritos urbanos, no han contribuido a generar procesos de exclusión social o funcional. En efecto, según la percepción de los habitantes de conjuntos de vivienda social de Angol no existen grandes problemas de acceso a los servicios ni funciones urbanas, ya que el reducido tamaño de la ciudad permite que todo se encuentre relativamente próximo a sus hogares, y si bien existen problemas en transporte y conexión en 
ciertos lugares de la ciudad, estos son más bien atribuibles a problemas de gestión en el transporte comunal y no a la propia concentración de la vivienda social. Finalmente, no fue posible evidenciar tampoco la existencia de estigmas territoriales hacia los conjuntos de vivienda social de la ciudad, tan comunes en otras ciudades de Chile, ya profundamente investigadas por la literatura nacional.

Las características espaciales de la segregación y la percepción del acceso a oportunidades de parte de los actores locales, explican en buena medida la tendencia a entender este problema social como un fenómeno “cuasi”natural y normal en las ciudades y sociedad chilena que señalan los tecnopolitas y habitantes de conjuntos de vivienda social de Angol. Sin embargo, entregan luces respecto a que el habitar cerca e integrado, no necesariamente se traduce en un aumento de la sociabilidad o vínculo social pluriclasista, ya que reconocen la existencia al interior de la sociedad local de "microambientes" definidos por la condición socio-económica, laboral y cultural que presentan poca permeabilidad. Esto confirma lo planteado por Saravi (2008) y Ruiz-Tagle \& López-Morales (2014) acerca de que el habitar en condiciones de proximidad espacial y en contexto de baja segregación espacial, no necesariamente significan buenas relaciones entre vecinos e integración social ya que las dimensiones objetivas y subjetivas de la segregación, tal como se evidenció, pueden operar con lógicas diferentes.

Finalmente, cabe preguntar si ¿'las ciudades menores son una oportunidad de estudio que puede contribuir a suavizar los niveles de segregación de las ciudades mayores? Los resultados de este trabajo evidencian que la naturaleza de la segregación socio-espacial en Angol, y a modo de hipótesis en otras ciudades pequeñas, presenta tendencias diferentes a la de las grandes ciudades. Sin embargo, su estudio resulta relevante no sólo para entender cómo es que las dinámicas desenvueltas en las últimas décadas a la luz del capitalismo neoliberal operan en este tipo de espacios urbanos, sino que también para potencialmente anticipar los efectos que podría tener algunos procesos que actualmente comienzan a operar en las grandes áreas metropolitanas y ciudades intermedias, como la reducción de la escala espacial de la segregación y la conformación de nuevos barrios de integración social. Ampliar la mirada hacia las ciudades menores generaría un conocimiento valioso, que más tarde se podría traducir en el diseño de políticas públicas que permitan mejorar la convivencia en nuestras ciudades tan quebradas socialmente. 
Polis, Revista Latinoamericana, Volumen 15, $N^{\circ}$ 44, 2016

\section{Notas}

${ }^{1}$ Utilizamos la expresión ciudades menores para definir a todas aquellas que posean menos de 99.999 según los criterios establecidos por el MINVU (2007).

${ }^{2}$ La literatura también ha empleado para medir segregación la variable tenencia de vehículos por jefe de hogar, sin embargo en esta investigación la excluimos porque debido a la masificación que ha experimentado en las últimas décadas la propiedad de medios de transporte motorizados. 


\section{Bibliografía}

Arteaga, N. (2005), “El periplo del trabajo y la pobreza en la zona metropolitana del Valle de Toluca (1950-2000): del desarrollo interno a la economía global”. En Álvarez, M (Comp.), Trabajo y producción de la pobreza en Latinoamérica y El Caribe: estructuras, discursos y actores (pp. 349-372), Consejo Latinoamericano de Ciencias Sociales, Buenos Aires.

Borsdorf, A., Sánchez, R., \& Marchant, C. (2008), “Aprendiendo de los errores. La necesidad de cambios a la política nacional de vivienda en ciudades intermedias Chilenas”, Scripta Nova, 12(270). Disponible en http:// www.ub.edu/geocrit/sn/sn-270/sn-270-51.htm

Briggs, X. (2001), “Ties that bind, bridge and constrain: Social capital and segregation in the American metropolis”, en International Seminar on Segregation and the City, Cambridge.

Casgrain, A. (2010), “La apuesta del endeudamiento en la política habitacional chilena”, Revista INVI, 25(68), 155-182.

Cornejo, C. (2012), "Estigma territorial como forma de violencia barrial: El caso del sector El Castillo”, Revista INVI, 27(76), 177-200.

Ducci, M. (2005), “La política de vivienda como instrumento de desintegración social. Efectos de una política de vivienda exitosa”. En Castillo, M \& Hidalgo, R. (Eds.), 1906/2006 cien años de políticas de vivienda en Chile (pp. 107-123), Universidad de Chile, Santiago.

Escobar, S. (2005), “Globalización, trabajo y pobreza: el caso de Bolivia”. En Álvarez, S. (Comp.). Trabajo y producción de la pobreza en Latinoamérica y El Caribe: estructuras, discursos y actores (pp. 57-69), Consejo Latinoamericano de Ciencias Sociales, Buenos Aires.

Flores, C. (2003), “Consecuencias de la segregación residencial: Teorías y métodos”. Centro de Políticas Públicas, Pontificia Universidad Católica de Chile.

Garín, A., Salvo, S., \& Bravo, G. (2009a), “Segregación residencial y políticas de vivienda en Temuco: 1992-2002”, Revista de geografía Norte Grande, (44), 113-128.

Ídem. (2009b), “Tendencias en la segregación residencial en Chile”, Revista de Ciencias Sociales, 15(3), 407-418.

Garrido, J., \& Astorga, C. (2013), “Segregación barrial, exclusión social y delitos en la ciudad de Temuco-Padre Las Casas”. En Lara, R. \& Gaete, A. (Eds.), Desviación social y delincuencia en Chile y en La Araucanía (pp. 135-157), Ediciones Universidad de La Frontera, Temuco. 
Polis, Revista Latinoamericana, Volumen 15, No 44, 2016

Greenstein, R., Sabatini, F. \& Smolka, M. (2000), “Segregación espacial urbana, consecuencias y respuestas normativas”, Land Lines, 12(6).

Hidalgo, R. (2005), La vivienda social en Chile y la construcción del espacio urbano en el Santiago del siglo XX. Instituto de Geografía, Santiago.

Ídem. (2007), “¿Se acabó el suelo en la gran ciudad?: Las nuevas periferias metropolitanas de la vivienda social en Santiago de Chile”, EURE, 33(98), 57-75.

Hidalgo, R., Zunino, H., \&Álvarez, L. (2007), “Emplazamiento periférico de la vivienda social en el Área Metropolitana de Santiago de Chile”, Scripta Nova, 11(245). Disponible en http://www.ub.edu/geocrit/sn/sn24527.htm

Lambiri, D., \& Vargas, M. (2011), Residential segregation and public housing policy, the case of Chile, Universidad Diego Portales, Santiago.

Lungo, M. \& Baires, S. (2001), Socio-Spatial Segregation and Urban Land Regulation in Latin American Cities, Lincoln Institute of Land Policy Conference Paper.

Martori, J., \& Hoberg, K. (2004), “Indicadores cuantitativos de segregación residencial: el caso de la población inmigrante en Barcelona”, Scripta Nova, 8(169). Disponible en http://www.ub.edu/geocrit/sn/sn-169.htm

Martori, J. C., Hoberg, K., \& Surinach, J. (2006), “Población inmigrante y espacio urbano: Indicadores de segregación y pautas de localización”, EURE, 32(97), 49-62.

MINVU (1976), Memoria 1975, Ministerio de Vivienda y Urbanismo, Santiago de Chile.

Ídem. (2007), Medición de la superficie ocupada por las ciudades de Chile de más de 15.000 habitantes: 1993 -2003, MINVU-Observatorio Urbano, Santiago.

Molina, I. (2001), “Segregación habitacional étnica en la ciudad sueca. Un proceso de racialización”, Scripta Nova, (90). Disponible en http:// www.ub.edu/geocrit/sn-90.htm

Molinatti, F. (2013), “Segregación residencial socioeconómica en la ciudad de Córdoba (Argentina): Tendencias y patrones espaciales”, Revista INVI, 28(79), 61-94.

Ortiz, J., \& Escolano, S. (2013), “Movilidad residencial del sector de renta alta del Gran Santiago (Chile): hacia el aumento de la complejidad de los patrones socioespaciales de segregación”, EURE, 39(118), 77-96. 
Peters, P. (2009), Spatial segregation in complex urban systems: housing and public policy in Santiago, Chile, The University of Texas at Austin, Austin.

Rodríguez J., \& Arriagada, C. (2004), “Segregación Residencial en la Ciudad Latinoamericana”, EURE 30(89), 5-24.

Rodríguez, A., \& Sugranyes, A. (2004), “El problema de vivienda de los” con techo”. EURE, 30(91), 53-65.

Ruiz-Tagle, J., \& López-Morales, E. (2014), “El estudio de la segregación residencial en Santiago de Chile: revisión crítica de algunos problemas metodológicos y conceptuales”. EURE,40(119), 25-48.

Sabatini, F. (2000), Segregación social en Santiago de Chile: conceptos, métodos y efectos urbanos, Pontificia Universidad Católica de Chile, Santiago.

Ídem. (2003), “La segregación social del espacio en las ciudades de América Latina”, Serie Azul, 35, 59-70.

Sabatini, F., \& Brain, I. (2008), “La segregación, los guetos y la integración social urbana: mitos y claves”, EURE, 34(103), 5-26.

Sabatini, F., \& Cáceres, G. (2004), "Los barrios cerrados y la ruptura del patrón tradicional de segregación en las ciudades latinoamericanas: el caso de Santiago de Chile”. En G. Cáceres \& F. Sabatini (Eds.), Los barrios cerrados en Santiago de Chile: entre la exclusión y la integración social, Instituto de Geografía, Santiago.

Sabatini, F., Cáceres, G., \& Cerda, J. (2001a), “Segregación residencial en las principales ciudades chilenas: Tendencias de las tres últimas décadas y posibles cursos de acción”, EURE, 27(82), 21-42.

Ídem. (2001b), “Segregación residencial en las principales ciudades chilenas: Tendencias de las tres últimas décadas y posibles cursos de acción”, EURE, 27(82), 21-42.

Sabatini, F., \& Salcedo, R. (2007), “Gated communities and the poor in Santiago, Chile: Functional and symbolic integration in a context of aggressive capitalist colonization of lower-class areas”, Housing Policy Debate, 18(3), 577-606.

Sabatini F., \& Sierralta, C. (2006), “Medición de la segregación residencial: meandros teóricos y metodológicos, y especificidad latinoamericana”. Documento de Trabajo $N^{\circ} 38$. Santiago: Instituto de Estudios Urbanos, Facultad de Arquitectura, Diseño y Estudios Urbanos de la Pontificia Universidad Católica de Chile. 
Polis, Revista Latinoamericana, Volumen 15, No 44, 2016

Salcedo, R., \& Torres, A. (2004), “Gated communities in Santiago: wall or frontier?”, International Journal of Urban and Regional Research, 28(1), 27-44.

Saraví, G. (2008), "Mundos aislados: segregación urbana y desigualdad en la ciudad de México”, EURE, 34(103), 93-110.

Simian, J. M. (2010), “Logros y desafíos de la política habitacional en Chile”, Estudios públicos, (117), 269-322.

Suárez, A. (2005), Segregación residencial y pobreza. Consecuencias del aislamiento social de residentes en asentamientos precarios. Ponencia presentada en el XXV Congreso de la Asociación Latinoamericana de Sociología, Porto Alegre.

Ugarteche, O. (2000), “Globalización y crisis en debate”. En Acosta, A. (Compilador), El desarrollo en la globalización. El reto en América Latina, Editorial Nueva Sociedad, Quito.

Valdés, E. \& Koch, M. (2009), “Tendencias de segregación residencial en metrópolis latinoamericanas intermedias al inicio del siglo XXI. Porto Alegre (Brasil) y Córdoba (Argentina)”, Revista Líder, 15(11), 85-104.

Vergara, L. (2014), “El estado subsidiario y sus políticas urbanas: la expulsión de los estratos bajos de la ciudad”, GeoGraphos, 5(62), 146-166.

Ídem (2015), “Globalización neoliberal y los cambios de una ciudad pequeña: el caso de Angol, Chile”. Estudios Sociales, 23(46), 9-32.

Vergara, L., \& Rozas, M. (2013), “La vivienda social en la estructura urbana de Angol, 1992-2012”, Anales de la Sociedad Chilena de Ciencias Geográficas, En prensa.

Zunino, H. (2006), “Power relations in urban decision-making: neo-liberalism, "techno-politicians” and authoritarian redevelopment in Santiago, Chile”, Urban Studies, 43(10), 1825-1846.

Recibido: 25.10 .14

Aceptado: 13.07.15 